\title{
Quality of life and functional results in canal wall down vs canal wall up mastoidectomy
}

\author{
Qualità della vita e risultati funzionali nella timpanoplastica aperta \\ vs timpanoplastica chiusa \\ D. LUCIDI, E. DE CORSO, G. PALUDETTI, B. SERGI \\ Fondazione Policlinico Universitario A. Gemelli IRCCS, Università Cattolica del Sacro Cuore, Rome, Italy
}

\section{SUMMARY}

Social functioning and personal satisfaction about quality of life are issues in the spotlight in most fields of otolaryngology. However, in ear surgery, few studies performed standardised measurements through interviews and validated questionnaires. We enrolled 81 patients undergoing tympanomastoidectomy from January 2011 to December 2014, at the "A. Gemelli” Hospital of the Catholic University, Rome. 50 patients (61.7\%) underwent non-obliterative Canal Wall Down (CWD) mastoidectomy, whereas 31 patients (38.3\%) underwent Canal Wall Up (CWU) mastoidectomy. We administered the Chronic Ear Survey (CES) 3 and 12-months post-operatively and the Chronic Otitis Media Outcome Test-15 (COMOT-15) 12 months post-operatively. Results were compared to hearing threshold, sex and age. In the CWD Group, significant improvements were observed in all CES subscale scores and total scores over time $(\mathrm{p}<0.001)$ whereas in the CWU Group we found a partial improvement. Inter-group comparison showed no significant differences in administration of CES in CWD vs CWU ( $p>0.05$ for all subsections and overall scores). A significant difference was found only in the COMOT-15 "Hearing Function" subsection, in favour of CWU over CWD (61 vs 39 respectively; p < 0.05). A significant association was found between PTA and COMOT-15 "Hearing Function" subsection scores. According to our results, a significant difference in the post-operative QoL between CWD and CWU should not be taken for granted.

KEY WORDS: Cholesteatoma $\bullet$ Middle ear $\bullet$ Mastoidectomy $\bullet$ Ear surgery $\bullet$ Quality of life $\bullet$ Canal wall up $\bullet$ Canal wall down $\bullet$ Chronic otitis media

\section{RIASSUNTO}

Il benessere sociale e la soddisfazione personale riguardo alla qualità di vita sono temi d'attualità nella maggior parte dei campi dell'otorinolaringoiatria. Tuttavia, nella chirurgia dell'orecchio, pochi studi utilizzano misurazioni standardizzate elo questionari convalidati. Abbiamo arruolato 81 pazienti sottoposti a timpanoplastica da gennaio 2011 a dicembre 2014, presso l'Università Cattolica di Roma, Policlinico "A. Gemelli”. 50 pazienti $(61,7 \%)$ sono stati sottoposti a timpanoplastica aperta non-obliterativa (CWD), mentre 31 pazienti (38,3\%) sono stati sottoposti a timpanoplastica chiusa (CWU). Abbiamo somministrato il questionario Chronic Ear Survey (CES) 3 e 12 mesi dopo l'intervento e il questionario Chronic Otitis Media Outcome Test-15 (COMOT-15) 12 mesi dopo l'intervento. I risultati dei tests sono stati correlati con la soglia uditiva, il sesso e l'età. Nel gruppo CWD sono stati osservati miglioramenti significativi dei punteggi di tutte le sottosezioni del CES e dei punteggi totali in funzione del tempo $(p<0,001)$, mentre nel gruppo CWU abbiamo riscontrato un parziale miglioramento. Il confronto tra timpanoplastica aperta e chiusa non ha mostrato differenze significative nella somministrazione del CES ( $p>0,05$ per tutte le sottosezioni e punteggi complessivi). Una differenza significativa è stata riscontrata solo nella sotto-sezione "Funzione uditiva" del COMOT-15, in favore del gruppo CWU (rispettivamente 61 versus 39; $p<0,05$ ). Una correlazione significativa è stata riscontrata solo tra i punteggi della sottosezione "Funzione uditiva" del COMOT-15 e il PTA. Secondo i nostri risultati, una differenza significativa nella qualità della vita postoperatoria nel confronto tra timpanoplastica aperta e chiusa non dovrebbe essere data per scontata.

PAROLE CHIAVE: Colesteatoma $\bullet$ Orecchio medio $\bullet$ Mastoidectomia $\bullet$ Chirurgia dell'orecchio $\bullet$ Qualità della vita $\bullet$ Timpanoplastica aperta $\bullet$ Timpanoplastica chiusa $\bullet$ Otite media cronica

\section{Introduction}

Cholesteatoma surgery represents one of the most debated topics in otolaryngology. The main goals are radical excision of the disease, prevention of severe complications due to the erosive behavior, possibly through the achievement of a dry and infection-free ear, and improvement of quality of life (QoL). Canal Wall Up (CWU) and Canal
Wall Down (CWD) mastoidectomy represent the most common surgical techniques. In CWU, the targets are achieved through preservation of both the external canal wall and middle ear volume and maintenance of a physiological position of the tympanic membrane. CWU allows to avoid both the need for frequent ear cleaning and the limitation of keeping the ear away from water. The majority of studies report better functional results obtained by 
CWU compared to CWD ${ }^{1-4}$. However, an increased risk of recurrence/residual cholesteatoma and revision surgery is described ${ }^{56}$. Moreover, the residual/recurrent cholesteatomatous process is not easily detectable in a CWU cavity. The literature reports varying recurrence rates in both groups. A recent review ${ }^{5}$, including six studies, describes higher recidivism after CWU (16.7-61\%) versus CWD (013.2\%). CWD allows easier outpatient follow-up and an early identification of cholesteatomatous foci or infection. On the other hand, in CWD some disadvantages may be present. The most common limitations are accumulation of keratin debris and need for frequent cleaning, higher susceptibility to infection with water exposure, risk of sudden dizziness associated with change of temperature in the external auditory canal and hearing aid discomfort ${ }^{7}$. To overcome such problems, many surgeons choose to obliterate the neo-cavity with different materials: bone patè, musculo-periosteal flaps, silicon material or cartilage, hydroxyapatite, or bioactive glass ${ }^{8-10}$.

Comparison of the two surgical techniques, in terms of post-operative outcomes, is mostly based on auditory results, complications and recurrence rates; however, the most common complaints among patients are impediment to social interaction and daily activity, due to frequent discharge from the ear, pain, medical examinations and fear of complications. Few studies have been designed to assess post-operative QoL in cholesteatoma patients and few specific tools are available. The first post-operative questionnaires administered to patients with chronic otitis media (COM), such as Glasgow Benefit Inventory (GBI), Short Form-36 (SF-36), Hearing Handicap Inventory for Adults (HHIA) and modified Amsterdam Inventory for Auditory Disability and Handicap Score (mAIAD), proved to be inadequate to evaluate the specific characteristic of these patients, because they are too generic or because only partial features, such as hearing loss, are evaluated. More recently, specific surveys aimed at the evaluation of specific aspects of COM and the impact on daily life have been developed ${ }^{11}$. The Chronic Ear Survey (CES) is a statistically validated questionnaire, specific for patients affected by COM, first introduced by Nadol and colleagues at the Massachusetts General Hospital ${ }^{12}{ }^{13}$, which has significant correlation with the audiometric threshold, with other QoL surveys (HHIA, SF-36), and undergoing to a marked post-surgical improvement. The Chronic Otitis Media Outcome Test-15 (COMOT-15) was subsequently developed and validated by Baumann et al. ${ }^{14}$ to allow self-assessment of symptom severity, whereas CES is based on symptom frequency.

The aim of our study was to assess the post-operative QoL in a population of patients operated for cholesteatoma. We compared the early and one year-postoperative results in CWD versus CWU without obliteration of the surgical cavity using the CES and COMOT-15 surveys. We finally compared subjective outcomes with age, sex and postoperative hearing threshold.

\section{Materials and methods}

We enrolled 81 patients admitted for cholesteatomatous otitis media at the Department of Head and Neck Surgery, Catholic University School of Medicine and Surgery, Rome, Italy. Patients underwent tympanomastoidectomy from January 2011 to December 2014. All enrolled patients showed recurrent otorrhoea and associated hearing loss and were treated with repeated cycles of antibiotics and corticosteroid therapy with poor improvement of the clinical picture. Micro-otoscopy and pure tone audiometry were performed in all patients. The diagnosis was confirmed by CT scan of the temporal bone, which showed inflammatory tissue in the middle-ear with partial erosion of adjacent bone structures.

All patients were candidates for surgery: in 50 patients (61.7\%) a CWD technique was performed, due to the large extent of the pathology, anatomical conformation and/or erosion of the external ear canal (CWD group). In 31 patients (38.3\%), we performed a CWU tympanomastoidectomy due to the limited extent of the disease (CWU group). In 5 patients, a sequential bilateral tympanoplasty was performed with analogous technique (3 CWD and 2 CWU). Standard surgery was performed under general anaesthesia, as an inpatient service, and in all cases a retroauricular incision with a tympanomeatal flap was made. We used temporal fascia for reconstruction of the tympanic membrane. CWU was performed in two stages, while CWD was carried out in a single stage. No obliteration of the neo-mastoid cavity was performed. Histological study confirmed a cholesteatomatous pattern in all cases, with keratinising squamous epithelium. All patients underwent standard pure-tone audiometry for testing conventional frequency range $(0.25$ to $8 \mathrm{kHz})$, using an Amplaid 319 audiometer (Amplaid Inc., Milan, Italy) in a double-walled, soundproof room. Pure-tone average (PTA) values were calculated as the mean of $0.5,1,2$, and $4 \mathrm{kHz}$ thresholds. Audiological assessment performed 24 hours preoperatively and 12 months post-operatively were compared.

Exclusion criteria were: patients younger than 18 years or older than 70 years, patients undergoing revision surgery, patients with bilateral disease who underwent a different surgical technique in the two ears, patients undergoing 
tympanoplasty without mastoidectomy, patients affected by petrous apex cholesteatoma and patients affected by severe general diseases influencing the degree of perceived quality of life or unable to fill out the questionnaire.

The Chronic Ear Survey questionnaire was assessed in all patients within the first 3 months after surgery (early post-operative administration). At one-year postoperative assessment (mean time after surgery $12.6 \pm 3$ months), all patients underwent a second administration of the Chronic Ear Survey together with the Chronic Otitis Media Outcome Test-15 (COMOT-15).

The Chronic Ear Survey is a 13-item survey, divided in 3 subscales: activity restriction (AR), symptoms ( $\mathrm{S}$ ) and medical resources (MR). The Chronic Otitis Media Outcome Test-15 (COMOT-15) consists of 15 items and 3 subscales: ear symptoms (ES), hearing function (HF) and mental health $(\mathrm{MH})$; in addition, a single question regarding a general evaluation on QoL (GE) and a single question on frequency of doctor visits (FDV) were included. Total scores were normalised in percentiles ( 0 100 , with 0 indicating maximum restriction of quality of life). This study was approved by the Ethics Committee of the Faculty of Medicine of the Catholic University of the Sacred Heart in Rome and was carried out in accordance with the Declaration of Helsinki. All patients received appropriate and comprehensible information about the surgical procedures and tests and gave their written consent. Statistical analysis of results was performed using SSPS for Windows. Continuous variables were expressed as mean \pm standard deviation (SD). Comparisons between groups were performed by Pearson's chi square test and Student's t-test. The strength of the correlation between the two parameters was obtained by Spearman's rank correlation test. The results were considered significant for $\mathrm{p}$ values $<0.05$.

\section{Results}

The mean age at the time of surgery was 47 years (range $=18-70 ; \mathrm{SD}= \pm 15.9)$ and the male/female ratio was 1.25 (45 males and 36 females). In the CWD group, the mean age was 48.5 years (range $=15-70 ; \mathrm{SD}= \pm 12.1$ ) and the male/female ratio was 1.3 (28 males and $22 \mathrm{fe-}$ males). In the CWU group, the mean age was 44.6 years (range $=15-70 ; \mathrm{SD}= \pm 16.8)$ and the male/female ratio was 1.2 (17 males and 14 females). The mean follow-up time was 22 months (range $=15-36$ months; $\mathrm{SD}= \pm 6$ ). At the time of data analysis, no patient was diagnosed with recurrent or residual cholesteatoma.

Pre-operatively, the overall mean PTA was $50 \mathrm{~dB}$ (range $=10-90 ; \mathrm{SD}= \pm 18$ ). In the CWD group, the mean pre-operative PTA was $55 \mathrm{~dB}$ (range $=16-90 ; \mathrm{SD}= \pm 18$ ), whereas in the CWU group the mean pre-operative PTA was $42 \mathrm{~dB}$ (range $=10-90 ; \mathrm{SD}= \pm 19$ ). The difference between the mean pre-operative PTA in the two groups was statistically significant $(\mathrm{p}<0.05)$. At the 12 -month post-operative assessment, the overall mean PTA was $48 \mathrm{~dB}$ (range $=10-90 ; \mathrm{SD}= \pm 19$ ). In the CWD group, the mean post-operative PTA was $52 \mathrm{~dB}$ (range $=22-81$; $\mathrm{SD}= \pm 18$ ), whereas in the CWU group the mean postoperative PTA was $41 \mathrm{~dB}$ (range $=16-65 ; \mathrm{SD}= \pm 18$ ). The difference between the mean post-operative PTA in the two groups was statistically significant $(\mathrm{p}<0.05)$. Significant differences between the pre- and post-operative PTA were not found $(p>0.05)$ in either the CWD or CWU groups. The pre- and post-operative audiological results of both groups are reported in Table I.

We compared the intra-group results obtained in the early (3 months) and late (12 months) post-operative administration of the Chronic Ear Survey. In the CWD group, significant improvements were observed in all the three mean subscale scores and total scores over time $(p<0.001)$. In the CWU group, we found a significant improvement in the mean activity restriction subscale score and overall score $(p<0.001)$ whereas the mean symptoms and medical resources subscale scores were not significantly different in the two administrations ( $p>0.05)$. The intra-group results of both groups are reported in Figure 1.

We compared the inter-group mean scores at both the early and late CES administration. At the early post-operative administration of CES, a significant difference was obtained only in the mean symptoms subscale score, in favour of the CWU group ( $<<0.05)$. However, at the late CES administration the mean subscale scores and total scores were not significantly different between groups $(p>0.05)$. Data regarding the early and late CES administration is summarised in Table II. The one-year post-operative administration of COMOT-15 shows the absence of a significant difference between the mean scores obtained in the CWD and CWU groups in the total scores and subsections, except for the hearing function subscale, in favor of the CWU group $(\mathrm{p}<0.05)$. The COMOT-15 results are shown in Table III. No significant difference was found in the administration of COMOT-15 and CES, according to sex ( $p>0.05$ in all subsections and overall scores). We performed linear regression analysis to evaluate the association between the post-operative hearing threshold (PTA) and both CES and COMOT-15 subsections and overall scores. A significant linear association was found only between the PTA and the COMOT-15 "hearing function" subsection scores ( $r$ coefficient $=0.721 ; p<0.005$; Fig. 2). Moreover, a significant correlation between the 
Table I. Pre- and post-operative audiological data in both groups.

\begin{tabular}{llccccc} 
& & $500 \mathrm{~Hz}$ & $1000 \mathrm{~Hz}$ & $2000 \mathrm{~Hz}$ & $4000 \mathrm{~Hz}$ & Mean PTA \\
CWD & Pre-op PTA (dB) \pm SD & $54 \pm 19^{*}$ & $55 \pm 19$ & $51 \pm 19^{*}$ & $60 \pm 21^{*}$ & $55 \pm 18^{*}$ \\
& Post-op PTA (dB) \pm SD & $48 \pm 19^{\S}$ & $51 \pm 19$ & $53 \pm 19^{\S}$ & $56 \pm 20$ & $52 \pm 18^{\S}$ \\
& Delta PTA (dB) \pm SD & $6 \pm 18$ & $4 \pm 20$ & $-2 \pm 18$ & $4 \pm 22$ & $3 \pm 19$ \\
CWU & Pre-op PTA (dB) \pm SD & $41 \pm 19^{*}$ & $44 \pm 19$ & $39 \pm 20^{*}$ & $45 \pm 21^{*}$ & $42 \pm 19^{*}$ \\
& Post-op PTA (dB) \pm SD & $35 \pm 19^{\S}$ & $43 \pm 19$ & $39 \pm 19^{\S}$ & $46 \pm 20$ & $41 \pm 18^{\S}$ \\
& Delta PTA (dB) \pm SD & $6 \pm 20$ & $1 \pm 18$ & $0 \pm 20$ & $-1 \pm 19$ & $1 \pm 18$ \\
\hline
\end{tabular}

Delta PTA: pre-operative minus post-operative PTA; " and ${ }^{\xi}: p<0.05$ for inter-group comparison.

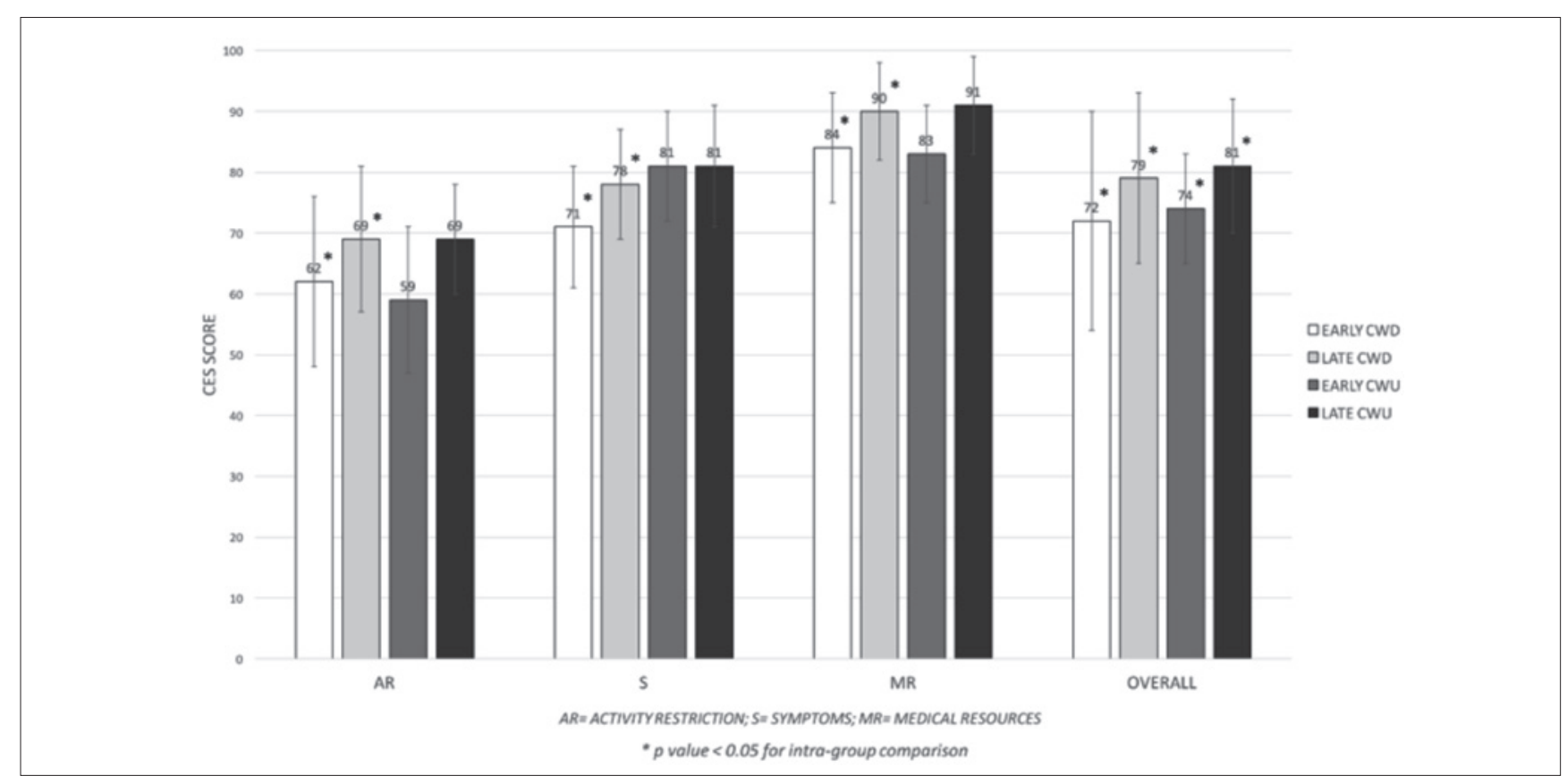

Fig 1. Comparison of 3-month (early) and 12-month (late) post-operative administration of CES in the CWD and CWU groups.

CES and COMOT-15 scores and age was not found ( $p>0.05$ in all the subsections and overall scores).

\section{Discussion}

Over the past 30 years, the concept of health-related quality of life has gradually gained large consensus to evaluate post-operative results in most fields of otolaryngology. Historically, CWD has been associated with a poorer quality of life, compared to CWU due to the limitations of the wide neo-mastoid cavity ${ }^{15}$. However, standardised measurements obtained through interviews and validated questionnaires are lacking.

In our study, we demonstrated the absence of a significant difference, in terms of self-perceived quality of life, in patients undergoing CWU compared to CWD. Our results are consistent with those reported by other authors ${ }^{912} 16$.
The study conducted by Lailach et al. ${ }^{16}$ which compared QoL outcomes among CWD, CWU and exclusively transcanalar technique (ETC) demonstrated that CWD showed similar results in QoL compared to CWU, whereas the ETC group reported significantly higher performances. All previous scientific comparisons, however, were conducted on CWD with mastoid obliteration versus CWU. Accordingly, it is suggested that the overlapping results are due to a decreased incidence of neo-mastoid cavity disadvantages in the CWD group ${ }^{891617}$.

Dornhoffer et al. ${ }^{18}$ reported that most patients subjected to revision surgery for a draining cavity (including mastoid obliteration and cartilage reconstruction of the tympanic membrane) have increased self-perceived QoL. Similarly, Kurien et al. ${ }^{7}$ suggested that secondary mastoid obliteration provides subjective benefit to patients, which is more 
Table II. Inter-group comparison of early and late CES scores.

\begin{tabular}{lcccccc} 
& & & AR & S & MR & Overall \\
Early administration & CWD & Mean score \pm SD & $62 \pm 14$ & $71 \pm 10 *$ & $84 \pm 9$ & $72 \pm 18$ \\
& CWU & Mean score \pm SD & $59 \pm 12$ & $81 \pm 9 *$ & $83 \pm 8$ & $74 \pm 14$ \\
Late administration & Delta CWU minus CWD & & -3 & 10 & -1 & 2 \\
& CWD & Mean score \pm SD & $69 \pm 12$ & $78 \pm 9$ & $90 \pm 8$ & $79 \pm 14$ \\
& CWU & Mean score \pm SD & $69 \pm 9$ & $81 \pm 10$ & $91 \pm 10$ & $81 \pm 11$ \\
& Delta CWU minus CWD & & 0 & 3 & 1 \\
\hline
\end{tabular}

AR: activity restriction; S: symptoms; MR: medical resources ( $p<0.05$ in comparison of symptoms subscale at early administration only).

Table III. Inter-group comparison of COMOT-15 scores.

\begin{tabular}{lccccccc} 
& ES & HF & MH & GE & FDV & Overall \\
CWD & Mean score \pm SD & $75 \pm 14$ & $39 \pm 12^{*}$ & $74 \pm 19$ & $44 \pm 22$ & $80 \pm 19$ & $64 \pm 20$ \\
CWU & Mean score \pm SD & $75 \pm 16$ & $61 \pm 18^{*}$ & $78 \pm 16$ & $34 \pm 22$ & $88 \pm 16$ & $60 \pm 20$ \\
Delta CWD minus CWU & & 0 & 22 & 4 & -10 & 8 & -4 \\
\hline
\end{tabular}

ES: ear symptoms; HF: hearing function; MH: mental health; GE: general evaluation on QoL; FDV: frequency of doctor visits ( $p<0.05$ in comparison of hearing function subscale only).

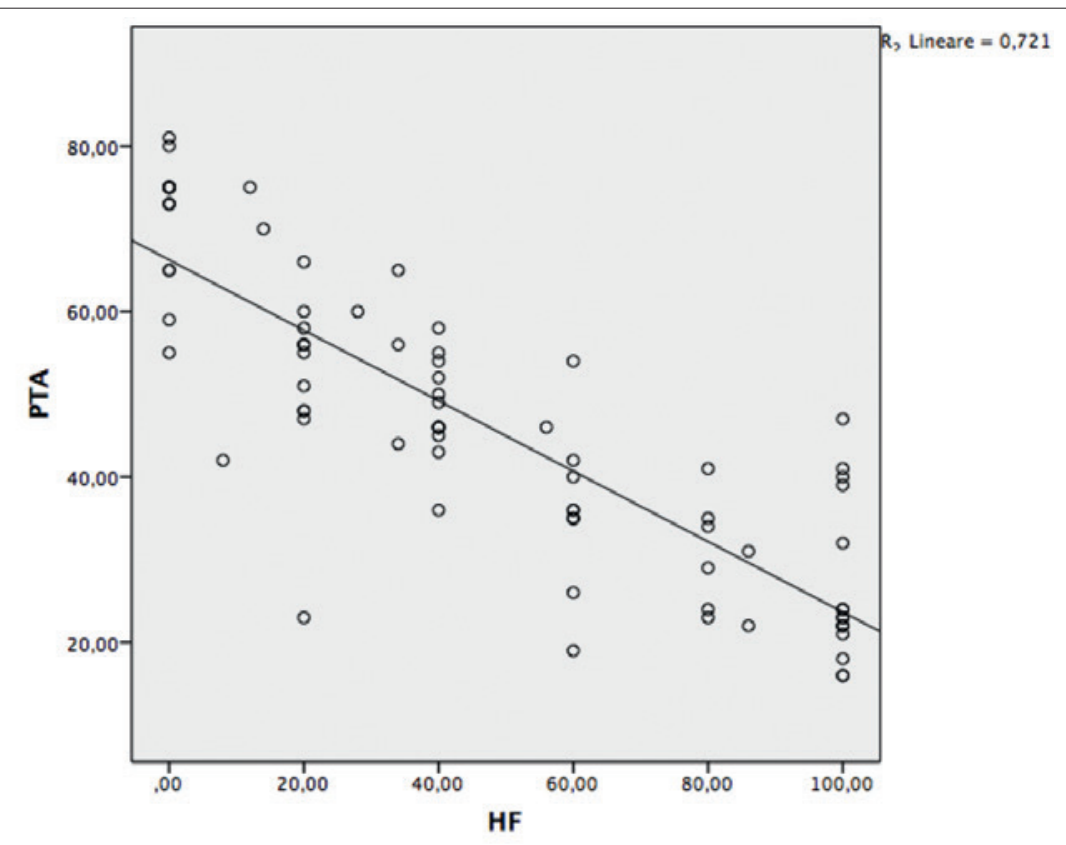

Fig 2. Scatter plot showing correlation between PTA levels (dB) and hearing function subsection scores at COMOT-15.

pronounced compared to primary mastoid obliteration. Both studies performed QoL assessment using the Glasgow Benefit Inventory (GBI), which is a generic tool for postsurgical evaluation and does not consider specific topics, such as otorrhoea, hearing loss, or water restriction. Moreover, the above-cited reports did not compare QoL obtained with different surgical techniques, but only analysed CWD patients undergoing obliteration of the mastoid cavity.
Our study is the first to demonstrate analogous results, in terms of QoL, in CWD with no mastoid obliteration versus CWU. Although promising long-term results following obliteration in CWD are described in the literature ${ }^{8}$, some critic aspects are reported by a recent overview ${ }^{19}$. Atrophy/resorption of muscle flaps, relapsing otorrhoea/ infection associated with reactive granulation tissue, often leading to surgical revision, are among the most fre- 
quently reported disadvantages. Moreover, a mild and not otherwise confirmed increase in the recurrence rate, associated with difficult exposure of the surgical cavity ("silent cholesteatoma" hidden in the obliterative tissue) is described ${ }^{819}$. We found that at 3-month post-operative assessment by CES, CWD patients reported a slightly lower mean score at the "symptoms" subsection (including the following entries: drainage, smell, hearing loss, pain), compared to CWU patients. This difference, however, was not statistically significant at the 12 -month post-operative assessment. Patients undergoing CWD mastoidectomy, in fact, tend to have delayed healing of the surgical cavity and higher initial psycho-social impairment compared to CWU patients, due to water restrictions and frequent postoperative medical examinations ${ }^{20}$. Moreover, the presence of exposed bone delays epithelisation of the mastoid bowl, leading to a higher risk of early post-operative otorrhoea.

However, in our experience, some precautions might be useful to accelerate the epithelial lining spread and to prevent otorrhoea, in the absence of obliteration of the mastoid cavity. We suggest avoiding bony overhanging in the hedges of the cavity and blind spots/pouches, to lower the facial ridge as much as possible, to remove all inflammatory tissue and to create an adequately sized meatoplasty. These measures allow satisfying epithelisation of the bowl in a few months ${ }^{21}$. This might explain the significant improvement reported by CWD patients in self-perception of ear symptoms over time. Accordingly, intra-group results obtained in comparison of early versus late administration of CES, demonstrated, in the CWD group, a significant improvement in all subsections (including "symptoms") and overall score. Together with ear-related symptoms, in CWD patients, distress related to the medical examinations and activity restriction (mainly water restrictions) becomes significantly less severe over time. On the other hand, in the CWU group, only "activity restriction" subsection and overall score significantly improved over time, whereas subjective assessment of distress related to "symptoms" and "medical resources" remained stable compared to the first trimester assessment. This is easily explained by the preservation of the physiological middle and external ear structure and the lower hospitalisation times. As reported by Nadol et al. ${ }^{12}$, perceived quality of life, assessed by CES scores, improves over time in patients subjected to tympanomastoidectomy, which is consistent with our results and, as previously described, is highly significant in CWD patients.

Overlapping outcomes were obtained by the administration of COMOT-15: only the "hearing function" subsection was characterised by significantly poorer results obtained by CWD versus CWU patients. Decreased auditory function remains the most disabling symptom experienced by patients undergoing to CWD compared to CWU ${ }^{22}{ }^{23}$. This is consistent with the poorer functional results obtained by this cohort. COMOT-15 assessment allowed us to demonstrate a significant difference between groups in terms of self-perceived hearing disability. It is, in fact, provided with a dedicated section. On the contrary, the CES questionnaire includes hearing discomfort in the symptoms subsection, together with drainage, smell and pain. COMOT-15 represents, in our opinion, a useful and complementary tool in assessment of quality of life in patients affected by COM, also thanks to the ease of administration (individual entries have 5 answer options each, whereas answer options vary from 4 to 6 in CES) and focus on symptom severity. Moreover, the COMOT-15 is provided with a specific section regarding mental health, which is not taken into account by the CES and which might be a critical issue in patients affected by chronic inflammatory disease and hearing impairment. Bakir and colleagues ${ }^{24}$ have shown a high prevalence of psychiatric symptoms such as depression, anxiety, phobia and somatisation in a population of patients affected by COM. Our results, however, do not demonstrate a high incidence of psychosomatic impairment or a significant difference in CWD versus CWU patients.

We compared post-operative hearing threshold and questionnaire scores, demonstrating that PTA $(\mathrm{dB})$ has only a linear correlation with the COMOT-15 "hearing function" subscale, thus confirming the validity of the test. However, no association was found between PTA levels and other CES and COMOT-15 subsection rates or overall scores. The relationship between audiometric threshold and questionnaire scores is a controversial topic: according to Nadol et al. ${ }^{12}$, the CES survey is specifically designed according to hearing levels and strong correlation between PTA and overall score is seen. Baumann et al. ${ }^{14}$ demonstrated a relationship only between hearing threshold and COMOT-15 HF and MH subscales. Lailach et al. ${ }^{16}$ demonstrated a moderate correlation between PTA and COMOT-15 overall score and strong correlation between PTA and the hearing function subscale. Other studies ${ }^{25}{ }^{26}$ showed only partial or no association at all between PTA and the questionnaire's subsections, consistent with our results. Our data imply that a patient with hearing impairment does not necessarily show subjective impairment in the overall quality of life. We believe that objective measurements, such as hearing threshold, are not sufficient to assess patient satisfaction. Other ear-related symptoms (such as smell, otorrhoea, pain, water restriction) and mental status are important 
in post-operative assessment for comparison of different techniques. Accordingly, administration of subjective assessment tests seems pivotal, in association with the collection of objective parameters, for post-operative assessment of COM patients.

This study has some limitations: CWD are performed in extensive cholesteatoma, and therefore patients may have a severe clinical outset. CWU patients often had small limited cholesteatoma with a mild pre-operative clinical picture. This might influence the early post-operative selfevaluation. All the studies comparing CWD versus CWU show this well-known limitation, since randomisation of the technique is not ethically acceptable.

Also, QoL results were collected on average 12 months post-operatively and although a longer follow-up time was administered for detection of cholesteatoma recurrence, this might only partially predict life-long results. We believe that one year after surgery epithelisation of the mastoid cavity is complete, allowing patients to provide a reliable self-assessment about quality of life. We also agree with the work by Nadol et al. ${ }^{12}$, who recognised that perceived quality of life improves over time and reaches its apex on the $12^{\text {th }}$ post-operative month. However, longterm results are also influenced by recurrence and a repeated surgical procedure could be considered reliable after at least 5 years follow-up.

The results in this study were collected in a limited cohort from a single institution and reproducibility was not demonstrated. Experience of the surgeon, social and cultural factors are crucial factors affecting outcomes. Potential confounders due to demographic features were avoided, since the two cohorts have consistent demographic characteristics, although the number of patients is not broad.

Moreover, the impact of recurrence and possible surgical reoperation on QoL could not be considered. In our institution, recurrence rates vary between $20-30 \%$ in CWU and $5-9 \%$ in CWD, in agreement with other reports ${ }^{5}$; however, at 22 months postoperative evaluation no patient enrolled in the study was diagnosed with recurrent cholesteatoma.

We believe that follow-up duration may not be sufficient to consider this result as conclusive. Indisputably, any future reoperation could compromise overall QoL, although at present it is not known if one group will be significantly more affected by recurrence than the other ${ }^{27}$.

\section{Conclusions}

Our data, although collected on a small sample, suggest that at one-year postoperative follow-up no differences exist in terms of quality of life between CWD with no mastoid obliteration and CWU surgeries. Long-term re- sults are needed, and we propose to address this important topic in a future study.

\section{Conflict of interest statement}

None declared.

\section{References}

1 Whittemore KR Jr, Merchant SN, Rosowski JJ. Acoustic mechanisms: canal wall-up versus canal wall-down mastoidectomy. Otolaryngol Head Neck Surg 1998;118:751-61.

2 Stankovic MD. Audiologic results of surgery for cholesteatoma: short- and long-term follow-up of influential factors. Otol Neurotol 2008;29:933-40.

3 Schmerber S, Troussier J, Dumas G, et al. Hearing results with the titanium ossicular replacement prostheses. Eur Arch Otorhinolaryngol 2006;263:347-54.

4 De Corso E, Marchese MR, Sergi B, et al. Role of ossiculoplasty in canal wall down tympanoplasty for middle-ear cholesteatoma: hearing results. J Laryngol Otol 2007;121:324-8.

5 Kerckhoffs KG, Kommer MB, van Strien TH, et al. The disease recurrence rate after the canal wall up or canal wall down technique in adults. Laryngoscope 2016;126:980-7.

6 Neudert M, Lailach S, Lasurashvili N, et al. Cholesteatoma recidivism: comparison of three different surgical techniques. Otol Neurotol 2014;35:1801-8.

7 Kurien G, Greeff K, Gomaa N, et al. Mastoidectomy and mastoid obliteration with autologous bone graft: a quality of life study. $\mathrm{J}$ Otolaryngol Head Neck Surg 2013;23:42-9.

8 Yamamoto Y, Takahashi K, Morita Y, et al. Long-term follow-up results of canal wall down tympanoplasty with mastoid obliteration using the bone pate plate for canal wall reconstruction in cholesteatoma surgery. Otol Neurotol 2014;35:961-5.

9 Quaranta N, Iannuzzi L, Petrone P, et al. Quality of life after cholesteatoma surgery: intact-canal wall tympanoplasty versus canal wall-down tympanoplasty with mastoid obliteration. Ann Otol Rhinol Laryngol 2014;123:89-93.

10 Bernardeschi D, Nguyen Y, Russo FY, et al. Cutaneous and labyrinthine tolerance of bioactive glass S53P4 in mastoid and epitympanic obliteration surgery: prospective clinical study. Biomed Res Int 2015;2015:242319.

11 Phillips JS, Yung MW. A systematic review of patient-reported outcome measures for chronic suppurative otitis media. Laryngoscope 2016;126:1458-63.

12 Nadol JB Jr, Staecker H, Gliklich RE. Outcomes assessment for chronic otitis media: the chronic ear survey. Laryngoscope 2000;110:32-5.

13 Ralli G, Milella C, Ralli M, et al. Quality of life measurements for patients with chronic suppurative otitis media: Italian adaptation of "Chronic Ear Survey". Acta Otorhinolaryngol Ital 2017;37:51-7.

14 Baumann I, Gerendas B, Plinkert PK, et al. General and diseasespecific quality of life in patients with chronic suppurative otitis media - a prospective study. Health Qual Life Outcomes 2011;9:48.

15 Bhatia S, Karmarkar S, DeDonato G, et al. Canal wall down mastoidectomy: causes of failure, pitfalls and their management. J Laryngol Otol 1995;109:583-9.

16 Lailach S, Kemper M, Lasurashvili N, et al. Health-related quality of life measurement after cholesteatoma surgery: comparison 
of three different surgical techniques. Eur Arch Otorhinolaryngol 2015;272:3177-85.

17 Clark MP, Bottrill I. SerenoCem-glass ionomeric granules: a 3-year follow-up assessment of their effectiveness in mastoid obliteration. Clin Otolaryngol 2007;32:287-90.

18 Dornhoffer JL, Smith J, Richter G, et al. Impact on quality of life after mastoid obliteration. Laryngoscope 2008;118:1427-32.

19 Alves RD, Cabral Junior F, Fonseca AC, et al. Mastoid obliteration with autologous bone in mastoidectomy canal wall down surgery: a literature overview. Int Arch Otorhinolaryngol 2016;20:76-83.

20 Bastier PL, Leroyer C, Lashéras A, et al. Early and late surgical site infections in ear surgery. Acta Otorhinolaryngol Ital 2016;36:127-34.

21 Thiel G, Rutka JA, Pothier DD. The behavior of mastoidectomy cavities following modified radical mastoidectomy. Laryngoscope 2014;124:2380-5.

22 Pontillo V, Barbara F, De Robertis V, et al. Treatment of cholestea- toma with intact ossicular chain: anatomic and functional results. Acta Otorhinolaryngol Ital 2018;38:61-6.

23 Artuso A, di Nardo W, De Corso E, et al. Canal wall down tympanoplasty surgery with or without ossiculoplasty in cholesteatoma: hearing results. Acta Otorhinolaryngol Ital 2004;24:2-7.

24 Bakir S, Kinis V, Bez Y, et al. Mental health and quality of life in patients with chronic otitis media. Eur Arch Otorhinolaryngol 2013;270:521-6.

25 Jung KH, Cho YS, Hong SH, et al. Quality-of-life assessment after primary and revision ear surgery using the chronic ear survey. Arch Otolaryngol Head Neck Surg 2010;136:358-65.

26 Choi SY, Cho YS, Lee NJ, et al. Factors associated with quality of life after ear surgery in patients with chronic otitis media. Arch Otolaryngol Head Neck Surg 2012;138:840-5.

27 Alicandri-Ciufelli M, Marchioni D, Kakehata S, et al. Endoscopic management of attic cholesteatoma: long-term results. Otolaryngol Clin North Am 2016;49:1265-70.

Received: November 10, 2017 - Accepted: August 3, 2018

How to cite this article: Lucidi D, De Corso E, Paludetti G, et al. Quality of life and functional results in canal wall down vs canal wall up mastoidectomy. Acta Otorhinolaryngol Ital 2019;39:53-60. https://doi.org/10.14639/0392-100X-2005

Address for correspondence: Daniela Lucidi, Fondazione Policlinico Universitario A. Gemelli IRCCS, Università Cattolica del Sacro Cuore, largo A. Gemelli 1, 00168 Rome, Italy. Tel. +39 06 30154439. Fax +39 06 30151194. E-mail: dani.lucidi@gmail.com 